\title{
Cooperative Pursuit with Voronoi Partitions
}

\author{
Zhengyuan Zhou ${ }^{\mathrm{a}}$, Wei Zhang ${ }^{\mathrm{b}}$, Jerry Ding ${ }^{\mathrm{c}}$, Haomiao Huang ${ }^{\mathrm{d}}$, Dušan M. Stipanoviće ${ }^{\mathrm{e}}$, Claire Tomlin ${ }^{\mathrm{f}}$ \\ ${ }^{a}$ Department of Electrical Engineering, Stanford University, Stanford, CA, 94305, USA. \\ ${ }^{b}$ Department of Electrical and Computer Engineering, Ohio State University, Columbus, OH, 43210, USA \\ ${ }^{c}$ Department of Electrical Engineering and Computer Sciences, University of California, Berkeley, CA, 94720, USA \\ ${ }^{d}$ Kuna Systems, CA, USA \\ ${ }^{e}$ Control and Decision Group, Coordinated Science Laboratory, University of Illinois, Urbana, IL, 61801, USA \\ ${ }^{f}$ Department of Electrical Engineering and Computer Sciences, University of California, Berkeley, CA, 94720, USA
}

\begin{abstract}
This work considers a pursuit-evasion game in which a number of pursuers are attempting to capture a single evader. Cooperation among multiple agents can be difficult to achieve, as it may require the selection of actions in the joint input space of all agents. This work presents a decentralized, real-time algorithm for cooperative pursuit of a single evader by multiple pursuers in bounded, simply-connected planar domains. The algorithm is based on minimizing the area of the generalized Voronoi partition of the evader. The pursuers share state information but compute their inputs independently. No assumptions are made about the evader's control strategies other than requiring the evader control inputs to conform to a speed limit. Proof of guaranteed capture is shown when the domain is convex and the players' motion models are kinematic. Simulation results are presented showing the efficiency and effectiveness of this strategy.
\end{abstract}

Keywords: pursuit-evasion games, Voronoi, cooperative pursuit

\section{Introduction}

This paper studies a multi-agent pursuit-evasion game, with a number of pursuers attempting to capture a single evader in a simply connected planar region. The pursuers' speeds are equal to or greater than that of the evader, and the objective is to find a successful cooperative strategy for the pursuers. Finding cooperation strategies among multiple agents in adversarial games can be challenging, as computing solutions over the joint state space of multiple agents can greatly increase computational complexity. The class of pursuit-evasion games considered here can, in a general theoretical setting, be treated as a multi-agent differential game. The solution to such a problem can, in principle, be obtained by solving the corresponding Hamilton-JacobiIsaacs (HJI) partial differential equation (PDE) [4, 11, 18, 21]. In particular, one can define the game through a value function representing the time-to-capture, with the evader attempting to maximize this function and the pursuers attempting to minimize this function. Under certain technical conditions, the value of the game can be characterized as the solution to an HJI equation, which can be in turn used to synthesize optimal controls for the pursuers to minimize time-to-capture. Solutions to HJI equations are typically found either using the method of characteristics $[4,18]$, in which optimal trajectories are found by

\footnotetext{
This work has been supported in part by NSF under CPS:ActionWebs (CNS-931843), by ONR under the HUNT (N0014-08-0696) and SMARTS (N00014-09-1-1051) MURIs and by grant N00014-12-1-0609, by AFOSR under the CHASE MURI (FA9550-10-1-0567).

Email addresses: zyzhou@stanford.edu (Zhengyuan Zhou), zhang@ece.osu.edu (Wei Zhang), jerryding@berkeley.edu (Jerry Ding), haomiao@stanf ord.edu (Haomiao Huang), dusan@illinois .edu (Dušan M. Stipanović), tomlin@eecs.berkeley.edu (Claire Tomlin)
}

integrating backward from a known terminal condition, or via numerical approximation of the value function on a grid of the continuous state space $[10,13,16,21]$.

The practical usage of the differential game approach, however, faces several computational challenges. While the characteristic solutions are useful in understanding optimal solutions qualitatively, they require backward integration from terminal configurations, which can make it difficult to generate strategies when only the initial configurations of the agents are known. On the other hand, HJI computation on grids suffers from the curse of dimensionality: computing solutions to HJI equations is computationally infeasible for scenarios with a large number of agents, as the grid required for approximating the value function grows exponentially in the dimensions of the joint configuration space.

For certain games and game configurations, it is possible to construct strategies for the agents geometrically. For example, pure-distance pursuit, in which a pursuer minimizes the instantaneous distance to the evader, has been shown to be the optimal pursuit strategy for certain zero-sum differential games in open environments [18], as well as a choice of strategy which guarantees capture in simply-connected regions [2]. In other cases, strategies based upon a geometric argument have also been found for coordinating groups of pursuers in open, unbounded spaces [19]. These methods are computationally efficient in generating control strategies, but are often limited to relatively simple game environments with no obstacles and typically assume homogeneous player speed.

In addition to games in continuous time and continuous spaces, research in discrete, turn-based games played on graphs have shown that three pursuers are sufficient and sometimes 
necessary to capture any evader in a planar graph [1, 22]. These results for discrete games have led to strategies for a class of continuous games known as visibility-based pursuitevasion $[14,15,20]$. The graph-based analysis has also recently inspired results for continuous games showing that three pursuers are also sufficient and sometimes necessary to capture an evader with equal speed in bounded polygonal domains with obstacles [6]. Similar to the graph and visibility pursuit strategies, they operate on the principle of successively reducing the game domain into a single simply-connected region by having pursuers block the evader from portions of the game space. However, to the best of our knowledge, currently implementable versions of these strategies do not yet exist. For all these methods, the game domain reduction requires searching over a large set of discrete actions, limiting the size of problems that can be practically solved.

In this paper, we present a decentralized pursuit strategy based on the Voronoi decomposition of the game domain with respect to agent positions, where the pursuers cooperatively minimize the area of the evader's Voronoi partition. Each pursuer influences the evader's partition only through the shared Voronoi boundary. Thus, each pursuer's input decouples from that of the other pursuers and can be computed independently. However, their inputs are coupled through the Voronoi partition, giving rise to cooperation among the pursuers. The pursuit algorithm is decentralized in the sense that the pursuers compute their control actions independently given the agent positions, which is the only shared information. This approach allows computation to take place in the low dimensional configuration space of individual agents instead of the high dimensional joint state space of all agents (as is the case in the HJI computation), thus enabling real-time implementation. We mention that the body of work described in [3,6] is relevant to the multiagent pursuit-evasion problems discussed in this paper, and is exciting as it provides formal proofs for the existence of pursuit strategies that guarantee capture, with provable bounds on the time-to-capture. On the other hand, the practical computation of such ideal strategies is still a subject of ongoing investigations. Our approach can be viewed as complementary to these theoretical contributions, in that we derive computable solution strategies for the particular problem scenario of convex environments and equal speeds. The insights obtained from this scenario may provide the foundation for the construction and implementation of more general strategies.

\subsection{Our Contributions}

Our contributions are threefold. First, we propose a Voronoi partition based pursuit strategy and show that, under the assumptions of convex environments, kinematic agent dynamics, and equal speeds of all players, this strategy results in guaranteed capture of the evader in finite time. Some elements of the results for convex domains and equal speeds were first presented in [17]. This work elaborates upon the previous results, gives an equivalent characterization of the control input that is more intuitive and easily implementable (Theorem 2) and provides a simpler proof of guaranteed capture based on a construction of an energy function (Theorem 3 ).
Second, we generalize the Voronoi pursuit strategy to nonconvex game domains and unequal agent speeds, in particular when the pursuers are faster than the evader, and show how to apply a modified fast marching method (FMM) [27] to quickly compute generalized Voronoi partitions and pursuer inputs. This approach provides a scalable, computationally efficient, and easily implementable algorithm for computing cooperative pursuer inputs in a multi-pursuer scenario.

Third, extensive simulation studies are carried out to show the effectiveness of the proposed strategy and compare its performance and computational complexity with several representative methods in the literature. In particular, it is shown that the algorithm encourages effective cooperative pursuit among multiple agents, resulting in superior performance to techniques such as the pure-pursuit, in which the pursuers attempt to minimize the instantaneous distance to the evader, and comparable performance to the optimal pursuit strategy based on HJI calculation, which is computational intensive and therefore not feasible beyond a single-pursuer-single-evader scenario.

\section{The Cooperative Pursuit Problem}

Consider a multi-agent pursuit-evasion game involving $N$ pursuers and a single evader, taking place in an open, simply connected region $\Omega$ in $\mathbb{R}^{2}$. Let $x_{e} \in \mathbb{R}^{2}$ be the position of the evader and $x_{p}^{i} \in \mathbb{R}^{2}$ be the position of pursuer $i$. The equations of motion are

$$
\begin{aligned}
\dot{x}_{e} & =d, x_{e}(0)=x_{e}^{0}, \\
\dot{x}_{p}^{i} & =u_{i}, x_{p}^{i}(0)=x_{p}^{i, 0}, i=1, \ldots, N,
\end{aligned}
$$

where $d$ and $u_{i}$ are the velocity control inputs of the evader and pursuers, respectively, and $x_{e}^{0}, x_{p}^{i, 0} \in \Omega$ are the initial evader and pursuer positions. The respective agent inputs are constrained to lie within sets $U_{i} \subset \mathbb{R}^{2}$ for the pursuers and $D \subset \mathbb{R}^{2}$ for the evader. In this paper, $U_{i}$ and $D$ are assumed to be the following:

$$
D=\left\{d \mid\|d\| \leq v_{e, \max }\right\}, U_{i}=\left\{u_{i} \mid\left\|u_{i}\right\| \leq v_{i, \max }\right\},
$$

where $v_{e, \max }$ and $v_{i, \max }$ are the maximum speeds of the evader and the pursuers, respectively, and $\|\cdot\|$ denotes the Euclidean norm in $\mathbb{R}^{2}$. The motions of the evader and pursuers, as described by equation (1), are also constrained to lie within the region $\Omega$, i.e.,

$$
x_{e}(t), x_{p}^{i}(t) \in \Omega, \forall t \geq 0 .
$$

Any velocity input $d(t)$ or $u_{i}(t)$ satisfying the constraints (2) and (3) is called an admissible input for the evader or pursuer $i$, respectively.

The goal of the pursuers is to capture the evader by having at least one of the pursuers come within a distance $r_{c}>0$ of the evader. To achieve this capture condition, each pursuer selects control inputs using a pursuit strategy $\mu_{i}\left(x_{e}, x_{p}^{1}, \ldots, x_{p}^{N}\right)$ based upon observations of the evader and pursuer positions at each time instant, which results in the closed-loop system dynamics:

$$
\begin{aligned}
\dot{x_{e}} & =d, x_{e}(0)=x_{e}^{0}, \\
\dot{x_{p}}{ }^{i} & =\mu_{i}\left(x_{e}, x_{p}^{1}, \ldots, x_{p}^{N}\right), x_{p}^{i}(0)=x_{p}^{i, 0}, i=1, \ldots, N .
\end{aligned}
$$


The evader may use some strategy $\gamma\left(x_{e}, x_{p}^{1}, \ldots, x_{p}^{N}\right)$ to avoid the pursuers. Any strategy $\mu_{i}$ satisfying the constraints (2) and (3) is called an admissible pursuit strategy for pursuer $i$. Admissible evasion strategy is defined similarly. The sets of admissible strategies for the pursuers and the evader are denoted by $\mathbb{U}$ and $\mathbb{D}$, respectively.

\section{Voronoi Based Pursuit in Convex Domain}

We begin our discussion of a pursuit strategy by focusing on an important class of the cooperative pursuit problem with a convex game domain and equal maximum speeds for all the players. Focusing on this special case enables us to develop a scalable and constructive solution to the cooperative pursuit problem, while also providing key insights into the solution of the general cooperative pursuit problem. In the rest of this section, we will first derive the proposed cooperative pursuit strategy and then show that it is guaranteed to capture the evader in finite time under all possible evader strategies. By appropriate re-scaling of problem parameters, our discussion assumes, without loss of generality, that the maximum speeds are $v_{e, \max }=v_{i, \max }=1, i=1, \ldots, N$.

\subsection{Voronoi-Based Cooperative Pursuit}

The pursuit strategy we propose is based on the Voronoi partition of $\Omega$ generated by the locations of the players. Roughly speaking, the strategy is designed so as to decrease the area of the evader's Voronoi cell over time. Intuitively, as this area decreases to zero, the capture condition will be satisfied. In this subsection, we will describe the pursuit strategy and some mathematical properties necessary for the proof of finite time capture.

Let $\mathcal{V}(\Omega)=\left\{\mathcal{S}_{e}, V_{1}, \ldots, V_{N}\right\}$ be the Voronoi partition of $\Omega$ generated by the points $\left\{x_{e}, x_{p}^{1}, \ldots, x_{p}^{N}\right\}$ :

$$
\begin{aligned}
\mathcal{S}_{e}= & \left\{y \in \Omega \mid\left\|y-x_{e}\right\| \leq\left\|y-x_{p}^{i}\right\|, \forall i \leq N\right\}, \\
V_{i}=\left\{y \in \Omega \mid\left\|y-x_{p}^{i}\right\| \leq\right. & \\
& \left.\min \left\{\left\|y-x_{e}\right\|,\left\|y-x_{p}^{j}\right\|\right\}, \forall j \neq i\right\}, i \leq N .
\end{aligned}
$$

Let $\mathcal{N}_{e}$ be the set of pursuer indices that are Voronoi neighbors of the evader, with cardinality $n_{e}$. The edge shared by $\mathcal{S}_{e}$ and $V_{i}, i \in \mathcal{N}_{e}$ is called the line of control of pursuer $i$ and is denoted by $B_{i}$, where $L_{i}$ is the length of $B_{i}$ (see Figure 1 ). We denote by $A$ the area of the Voronoi cell $\mathcal{S}_{e}$ containing the evader. It can be easily verified that the area $A$ depends only on the locations of the neighboring pursuers and that this dependence is smooth whenever the pursuer locations are in $\Omega$. The time derivative of $A$ is given by

$$
\frac{d A}{d t}=\frac{\partial A}{\partial x_{e}} \dot{x}_{e}+\sum_{i=1}^{N} \frac{\partial A}{\partial x_{p}^{i}} \dot{x}_{p}^{i}
$$

We propose a cooperative pursuit strategy that jointly minimizes $\frac{d A}{d t}$. According to (5), this joint objective can be decoupled into the individual objectives of minimizing $\frac{\partial A}{\partial x_{p}^{i}} \dot{x}_{p}^{i}$ for each pursuer $i$. Since $A$ depends only on the Voronoi neighbors of the evader, we have $\frac{\partial A}{\partial p^{i}}=0$ for all $i \notin \mathcal{N}_{e}$. Thus, for any pursuer $i$ which is not a Voronoi neighbor of the evader, we simply set $\mathbf{u}_{i}=\frac{x_{e}-x_{p}^{i}}{\left\|x_{e}-x_{p}^{i}\right\|}$. On the other hand, for each pursuer $i \in \mathcal{N}_{e}$, the choice of pursuit strategy which minimizes (5) is given by:

$$
\mathbf{u}_{i}^{*}=\mu_{i}^{*}\left(x_{e}, x_{p}^{1}, \ldots, x_{p}^{N}\right) \triangleq \frac{-\frac{\partial A}{\partial x_{p}^{i}}}{\left\|\frac{\partial A}{\partial x_{p}^{i}}\right\|},
$$

where we make use of the assumption that $v_{\max }=1$.

Define $D_{h}^{i} A$ and $D_{v}^{i} A$ as the directional derivatives of $A$ along the unit vectors $\boldsymbol{\eta}_{h}^{i}$ and $\boldsymbol{\eta}_{v}^{i}$ (see Figure 1), then

$$
\left\{\begin{array}{l}
\left.D_{h}^{i} A\right|_{\left(x_{e}, x_{p}^{1}, \ldots, x_{p}^{N}\right)}=\lim _{\epsilon \rightarrow 0} \frac{\left.A_{i}^{+}\left(\epsilon \cdot \eta_{h}^{i}\right)\right|_{\left(x_{e}, x_{p}^{1}, \ldots, x_{p}^{N}\right)}-A}{\epsilon} \\
\left.D_{v}^{i} A\right|_{\left(x_{e}, x_{p}^{1}, \ldots, x_{p}^{N}\right)}=\lim _{\epsilon \rightarrow 0} \frac{\left.A_{i}^{+}\left(\epsilon \cdot \boldsymbol{\eta}_{v}^{i}\right)\right|_{\left(x_{e}, x_{p}^{1}, \ldots, x_{p}^{N}\right)}-A}{\epsilon}
\end{array}\right.
$$

where $A\left(x_{e}, x_{p}^{1}, \ldots, x_{p}^{N}\right)$ is denoted by $A$ for brevity. From this expression, the partial derivative of $A$ with respect to $x_{p}^{i}$ is then:

$$
\frac{\partial A}{\partial x_{p}^{i}}=D_{h}^{i} A \cdot \boldsymbol{\eta}_{h}^{i}+D_{v}^{i} A \cdot \boldsymbol{\eta}_{v}^{i} .
$$

The directional derivatives can be computed by the following lemma, whose proof can be found in [17].

Lemma 1. For any $i \in N_{e}$, it holds that

$$
D_{h}^{i} A=-\frac{L_{i}}{2}, D_{v}^{i} A=\frac{l_{i}^{2}-\left(L_{i}-l_{i}\right)^{2}}{2\left\|\xi_{i}\right\|} .
$$

Theorem 2. Under the proposed pursuit strategy given in Equation 6, pursuer $i$ should always head for the midpoint of the line of control $B_{i}$.

Proof. By Lemma 1, the control input is the vector $\left(D_{h}^{i} A, D_{v}^{i} A\right)$ in the local coordinate system ${ }^{1}$ defined by $\left\{\boldsymbol{\eta}_{h}^{i}, \boldsymbol{\eta}_{v}^{i}\right\}$. Let $\alpha$ be the angle between the velocity input and the local horizontal axis defined by $\boldsymbol{\eta}_{h}^{i}$. It is true that

$$
\tan (\alpha)=\frac{D_{v}^{i} A}{D_{h}^{i} A}=\frac{\frac{l_{i}^{2}-\left(L_{i}-l_{i}\right)^{2}}{2\left\|\xi_{i}\right\|}}{-\frac{L_{i}}{2}}=-\frac{2 l_{i}-L_{i}}{\left\|\xi_{i}\right\|} ;
$$

Let $\beta$ be the angle between the local horizontal axis and the vector from pursuer $i$ to the midpoint of $B_{i}$. Then

$$
\tan (\beta)=\frac{l_{i}-\left(L_{i} / 2\right)}{\left\|\xi_{i}\right\| / 2}=-\frac{2 l_{i}-L_{i}}{\left\|\xi_{i}\right\|} ;
$$

Therefore $\alpha=\beta$, thus establishing the theorem.

\subsection{Proof of guaranteed capture}

In this section, we will show that the pursuit strategy outlined above for the convex and equal speed game is guaranteed to capture the evader in finite time, regardless of any admissible evader input $d$. It can be seen that if this holds for the case of a

\footnotetext{
${ }^{1}$ See [17] for more details.
} 


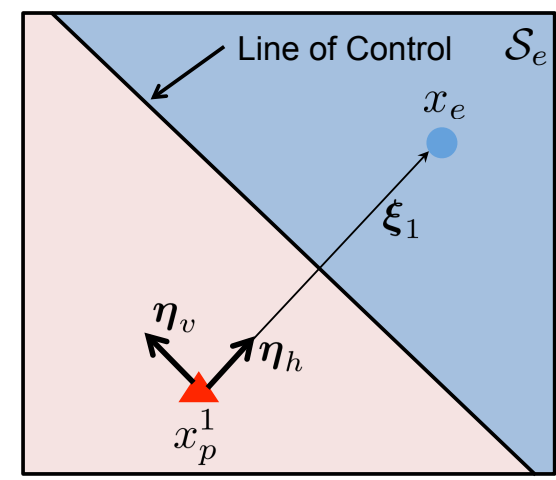

(a)

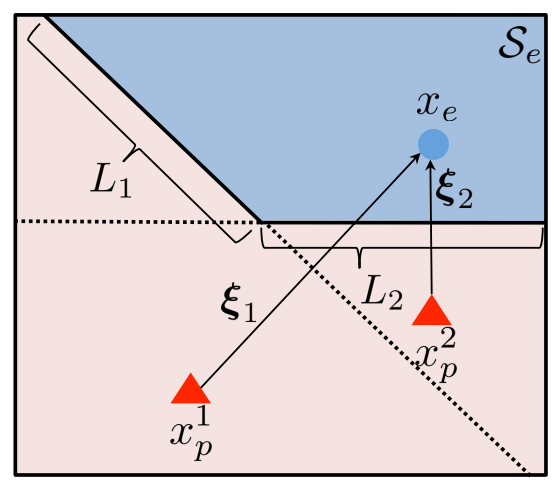

(b)

Figure 1: Illustrations showing the evader's Voronoi cell $\mathcal{V}_{e}$ in a convex domain with equal agent speed (a) for a single pursuer and evader; (b) with an additional pursuer.

single pursuer $(N=1)$, then the conclusion also extends to the case of multiple pursuers $(N>1)$. Indeed, for the case of $N>$ 1 , one can choose any pursuer $i$ which is a Voronoi neighbor of the evader and use the arguments for the case of $N=1$ to show that the capture condition will be satisfied. This section presents the proof for a single pursuer. Correspondingly, the notation from above will carry through without the index $i$.

We mention that although it is well-known that one pursuer is enough to guarantee capture by, for instance, specializing the pursuit strategies in prior work $[3,6]$ to the convex domain here, our argument in establishing the proof of guaranteed capture for the Voronoi-based cooperative pursuit strategy is novel. In particular, our proof technique is rather distinct from the prior work dealing with general domains ([3, 6]), as the arguments there typically start by showing that certain paths are guardable (i.e., evaders, after a finite amount of time, will never be able to cross it without getting captured) and proceed to successively shrink the safe domain for the evader using such guardable paths until capture is established. In our proof, we design an energy function, tailored specifically to the properties of our cooperative pursuit strategy, that is shown to always decrease over time at a linear rate, thereby ensuring that energy will decrease to 0 in finite time, at which point capture must happen. We now formalize this argument.

Consider an "energy" function $E=k A+z$ for a positive constant $k$ (to be defined subsequently), where $z(t)=\| x_{e}(t)-$ $x_{p}(t) \|_{2}^{2}$. Clearly, $E=0$ if and only if $A=0$ and $z=0$, both of which imply that capture occurs. A proof will now be presented that $E$ will decrease towards zero as $t$ increases.

Theorem 3. Under the pursuit strategy $\mu^{*}$ given in Equation 6, if the capture condition has not been met before time $t>0$, then for some positive constants $k, \beta, c(\beta)$ :

$$
E(t) \leq E(0)-c(\beta) t
$$

where $E(0)$ is the initial energy.

Proof. By Lemma 4 and Lemma 5 in [17], one of the following conditions must be true at any given time:

1. $\dot{A} \geq-\beta$ and $\dot{z} \leq-c(\beta)$, or

\section{2. $\dot{z}>c(\beta)$ and $\dot{A}<-\beta$.}

Note that the derivative of $E$ is $\dot{E}=k \dot{A}+\dot{z}$ and $\dot{A} \leq 0$ for all time. Then, under condition $1, \dot{A} \leq 0$ and $\dot{z} \leq-c(\beta)$, thus $\dot{E} \leq-c(\beta)$. The rate of change of $z$ is limited by the maximum speed of the two agents and the geometry of the domain. Since $\dot{z}=2 \delta \dot{\delta}, \delta \leq l_{\max }$, and $\dot{\delta} \leq 2$, then $\dot{z} \leq 4 l_{\text {max }}$. Now, let $k=$ $\frac{4 l_{\max }+c(\beta)}{\beta}$. Under condition $2, \dot{A}<-\beta$ and of course $\dot{z} \leq 4 l_{\text {max }}$, thus $\dot{E} \leq-k \beta+4 l_{\text {max }}$, therefore again $\dot{E} \leq-c(\beta)$, guaranteeing that the energy will decrease to 0 in finite time, and thus leading to capture.

\subsection{Advantages of Proposed Pursuit Strategy}

It is important to point out several distinctive features of the proposed Voronoi-based pursuit strategy. First, the strategy adopts a simple analytical expression in terms of the player locations, which enables fast online implementation of the strategy. Second, we have shown that the Voronoi-based strategy guarantees capture even with only one pursuer, which distinguishes the proposed strategy from many other heuristic-based pursuit strategies. The strategy also leads to effective cooperation among the pursuers that can dramatically reduce the capture time (see Section 5 for numerical illustrations). The reason behind the induced cooperation can be partly seen from the illustration in Fig. 1. Each pursuer affects $\mathcal{S}_{e}$ only through its shared boundary with the evader (line of control $L_{1}$ and $L_{2}$ in the figure). The derived Voronoi based strategy essentially allows each pursuer to push its line of control to the maximal extent along the most effective direction. Finally, the strategy also properly incorporates environment geometries into the control input design for the pursuers. The boundaries of the game domain provide additional line of controls as if there were more pursuers. Therefore, the pursuit strategy naturally includes constraints created by the environment.

\section{Extensions to Nonconvex Domains With Unequal Speeds}

In this section we extend the area minimization strategy to more realistic pursuit-evasion scenarios with non-convex game domains and unequal maximum speeds. The main challenge 
lies in the extension of the Voronoi partition concept to handle these realistic conditions. The desired extension requires a definition of Voronoi partitions that is independent of the specific game domain and player dynamics under consideration. Such decompositions in pursuit-evasion games have been considered for non-convex game domains when the pursuers and evader have equal speeds [9] and for convex domains with un-equal speeds [5]. We will examine the combined case of un-equal speeds and non-convex domains. For this purpose, we shall think of the evader's generalized Voronoi cell as a set of all the points that the evader can reach without being captured by any of the pursuers for all admissible pursuit strategies. We call this set the Safe Reachable Set and denote it by $\mathcal{S}_{e}$. The rest of this section will provide a formal definition of the safe reachable set and discuss its numerical computation as well as its applications in cooperative pursuit problems.

\subsection{Safe-Reachable Set}

Given initial conditions $x_{e}^{0}, x_{p}^{i, 0}$, a point $y \in \Omega$ is safereachable by the evader if there exists some $\gamma \in \mathbb{D}$ and $t \geq 0$ such that $x_{e}(t)=y$ and $x_{e}(s) \notin C(s)$ for all $s \in[0, t]$ and all $\mu_{i} \in \mathbb{U}$, where $C(s)$ is the set of all points that any pursuer can reach (counting the endowed capture radius) within time $s$. The safe-reachable set $\mathcal{S}_{e}$ of the evader is then defined as

$$
\mathcal{S}_{e}=\{y \in \Omega \mid y \text { is safe-reachable }\} .
$$

Define the minimum time-to-reach function $\varphi: \Omega \rightarrow \mathbb{R}$ for the evader constrained to $\mathcal{S}_{e}$ as follows :

$$
\varphi(y)=\min \left\{t \mid x_{e}(t)=y, x_{e}(s) \in \mathcal{S}_{e}, \forall s \in[0, t]\right\} .
$$

Similarly, a minimum time-to-capture function $\psi^{c}: \Omega \rightarrow \mathbb{R}$ for a pursuer in $\Omega$ is defined as:

$$
\psi^{c}(y)=\min \left\{t \mid y \in C(t), x_{p}(s) \in \Omega, \forall s \in[0, t]\right\} .
$$

The minimum time-to-capture function represents, for a point $y$, the minimum time required for the pursuer to capture the evader if the evader were stationary at $y . \mathcal{S}_{e}$ relative to a single pursuer is therefore

$$
\mathcal{S}_{e}=\left\{y \mid \varphi(y)<\psi^{c}(y)\right\} .
$$

For multiple pursuers, the evader must reach a point $y$ before all pursuers. Let $\psi_{i}^{c}$ be the minimum time-to-capture function for pursuer $i$. The evader must reach the point $y$ in less time than the minimum of all of the time-to-capture functions. Therefore

$$
\mathcal{S}_{e}=\left\{y \mid \varphi(y)<\psi_{i}^{c}(y), \forall i\right\} .
$$

Observe that the definitions of $\mathcal{S}_{e}$ and $\varphi$ are interrelated. Computing $\mathcal{S}_{e}$ requires simultaneously computing the set $\mathcal{S}_{e}$ and the values of $\varphi$ within that set. When the pursuer and evader speeds are equal, the safe-reachable set is equivalent to the evader's Voronoi cell in the generalized Voronoi decomposition of the agents [9]. In more complex situations the appropriate timeto-reach values can be computed using modified fast marching methods (FMM) as described in [27]. FMM [12, 23, 24] is a single-pass method used to numerically approximate the
Eikonal equation, which for the agent dynamics described here can be used to compute the time-to-reach and time-to-capture functions. In general, for kinematic agents with limited speeds $\mathcal{S}_{e}$ depends only on the position of the agents.

It should be noted that $\mathcal{S}_{e}$ is defined in the open-loop sense, in that a point in $\mathcal{S}_{e}$ can be reached by the evader moving directly toward that point and ignoring the actions of the pursuers. Furthermore, the computational efficiency of the proposed control strategy directly results from the fact that $\mathcal{S}_{e}$ is defined in the open-loop sense. On the other hand, the feedback nature of the strategy comes from the receding horizon implementation of the open-loop controls. Namely, the minimization of the open-loop safe reachable set is carried out at each time instant, given the current measurement of system state, thus allowing the pursuers to react to the movements of the evader. $\mathcal{S}_{e}$ is used by the pursuers to guide their pursuit strategy but does not necessarily affect the actions of the evader itself.

\subsection{Safe-Reachable Set Based Cooperative Pursuit}

With the safe reachable set introduced previously, we can now extend the Voronoi-based pursuit strategy to address the general cooperative pursuit problem. We adopt a similar idea that tries to minimize the area of the safe reachable set to progressively limit the evader's future safe locations. With slight abuse of notations, we let $A$ denote the area of $\mathcal{S}_{e}$. The time derivative of $A$ remains the same as in (5). A pursuer $i$ is said to share an active boundary with the evader if there is a portion of the boundary of $\mathcal{S}_{e}$ where $\psi_{i}^{c}(y)<\psi_{j}^{c}(y)$ for all points $y$ along this portion and all other pursuers $j$. In other words, there is a portion of the boundary of $\mathcal{S}_{e}$ that is defined purely by the position of pursuer $i$. This is analogous to having a shared Voronoi boundary with the evader in a Voronoi decomposition.

Let $N_{e}$ be the set of all pursuers that share an active boundary with the evader. The area $A$ only depends on the pursuers in $N_{e}$, so $\frac{\partial A}{\partial x_{p}^{i}}=0$ for all $i \notin N_{e}$. Following a similar idea as in Section 3 , any pursuer $i \notin N_{e}$ will simply use the pure-distance pursuit strategy. On the other hand, for each pursuer $i \in N_{e}$, the choice of pursuit strategy which minimizes $\frac{\partial A}{\partial x_{p}^{i}}$ is given by:

$$
\mathbf{u}_{i}^{*}=\mu_{i}^{*}\left(x_{e}, x_{p}^{1}, \ldots, x_{p}^{N}\right) \triangleq-v_{i, \max } \frac{\frac{\partial A}{\partial x_{p}^{i}}}{\left\|\frac{\partial A}{\partial x_{p}^{i}}\right\|} .
$$

Note that in the case of non-convex domains it may be possible for singular surfaces to arise where multiple inputs result in the same area derivative. In this case, the pursuer may simply choose the input maximizing the decrease in distance to the evader.

While an analytic solution was found for convex domains with equal agents' speeds, it is difficult to construct the safereachable set geometrically for the general case. Nevertheless, the modified fast-marching method (FMM) presented in [27] can be used to compute the safe-reachable set on a grid. The area of this set can be then used for numerical approximation of the pursuit strategy.

The details of the modified FMM algorithm can be found in [27] and will not be repeated here. A sketch of the algorithm 


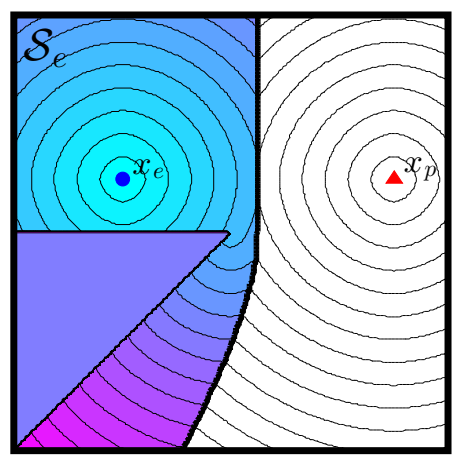

Figure 2: An example of safe-reachable set computed in a non-convex game domain, with contours plotted for equal time-to-reach values for each agent.
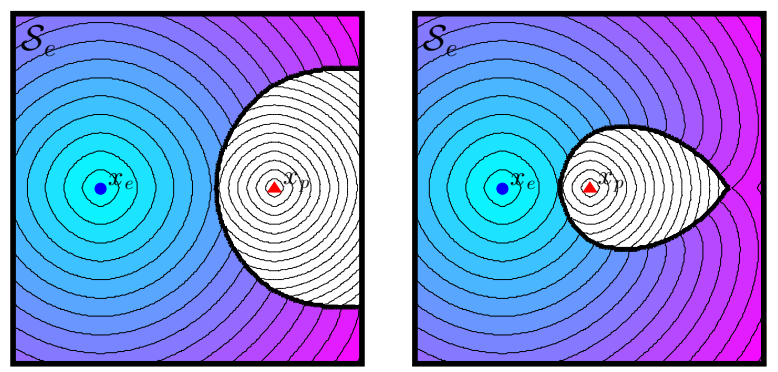

Figure 3: An illustration of a scenario where the pursuer (red triangle) is slower than the evader (blue circle). Note how $\mathcal{S}_{e}$ grows larger as the pursuer gets closer to the evader.

is as follows. First, the standard FMM algorithm is used to compute the time-to-capture $\psi_{i}^{c}\left(y_{j, k}\right)$ for every node $y_{j, k}$ on the grid. The modified FMM is then used to compute the safe-reachable set $\mathcal{S}_{e}$, beginning with the current position of the evader $x_{e}$, and successively adding points that can be reached in time $\varphi\left(y_{j, k}\right)$ with $\varphi\left(y_{j, k}\right)<\psi_{i}^{c}\left(y_{j, k}\right), \forall i$. An example of the computation performed for a non-convex region with a triangular obstacle and point capture $\left(r_{c}=0\right)$ is shown in Figure 2, with equal time-toreach values plotted for the two agents. Note that the boundary of $\mathcal{S}_{e}$ is no longer a straight line, since the evader must first move to the corner of the obstacle in order to reach points in the lower portion of the game domain.

Let $N_{s}$ be the number of grid nodes for which $\varphi\left(y_{j, k}\right)<$ $\psi_{i}^{c}\left(y_{j, k}\right), \forall i$. The area of $\mathcal{S}_{e}$ can be approximated as

$$
A \approx N_{s} h^{2}
$$

where $h$ is the grid spacing. The gradient with respect to pursuer movements can be numerically approximated by perturbing each pursuer by $h$ horizontally and vertically on the grid and recomputing $A$. The pursuit strategy is identical, with

$$
\mathbf{u}_{i}^{*}=\mu_{i}^{*}\left(x_{e}, x_{p}^{1}, \ldots, x_{p}^{N}\right) \triangleq-v_{i, \max } \frac{\frac{\partial A}{\partial x_{p}^{i}}}{\left\|\frac{\partial A}{\partial x_{p}^{i}}\right\|} .
$$

There are several things to note about using safe-reachable area pursuit in non-convex domains with unequal speeds. The first is that the algorithm is only effective in cases where the

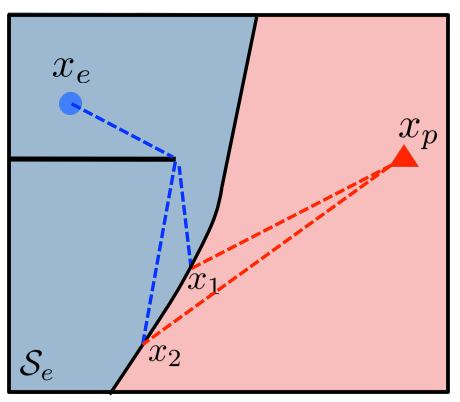

Figure 4: Illustration of the asymmetry between the evader and the pursuer when the domain is non-convex.

pursuers are at least as fast as the evader. This is due to the fact that, if the pursuer is slower than the evader, the evader's safe-reachable set may shrink in area as the pursuer moves farther away from the evader, as shown in Figure 3. Thus area minimization will likely not lead to capture.

A second point is that under the safe-reachable area minimization pursuit strategy, currently no proof has been found to guarantee capture in the non-convex case, although the area minimization strategy works well empirically. In a convex domain with faster pursuers, the guarantee of capture from the previous section holds trivially, for example if the pursuer simply matches the evader's speed and uses the equal speed algorithm. The major obstacle in the non-convex case is that the game is no longer symmetric with respect to the area of $\mathcal{S}_{e}$. This is illustrated in Figure 4, which shows the safe-reachable set for an evader that must turn around a non-convex obstacle. This configuration gives an advantage to the evader, as it moves in the same direction whether it is headed for the point $x_{1}$ or $x_{2}$. Thus, if the evader were to move distance $\epsilon$ toward $x_{1}$, it has also decreased its distance to $x_{2}$ by $\epsilon$. If the pursuer moves to maintain $x_{1}$ on the boundary of the safe-reachable set, then it can move $\epsilon$ toward $x_{1}$, but it will have moved some distance less than $\epsilon$ toward $x_{2}$, and $x_{2}$ will have become part of the evader's safe-reachable set. In this manner, the evader is able to increase the area of its safe-reachable set regardless of the pursuer input.

However, an advantage of this approach is that the safereachable set is defined without explicit dependence on the dynamics of the agents. For example, although simple kinematic dynamics are considered here, it is possible to use FMM formulations to compute reachable sets for more complex dynamics such as the Dubin's car [25]. The computational approach also lends itself well to cases with spatially-varying velocity profiles, where the maximum speeds of the agents may change depending on position [27]. Thus the pursuit algorithm can be used whenever the safe-reachable set of the evader can be computed in an efficient manner.

\section{Simulation Results}

A number of simulations were conducted to evaluate the proposed safe-reachable area minimization pursuit strategy. The performance of the pursuit strategy was compared in a sequence 

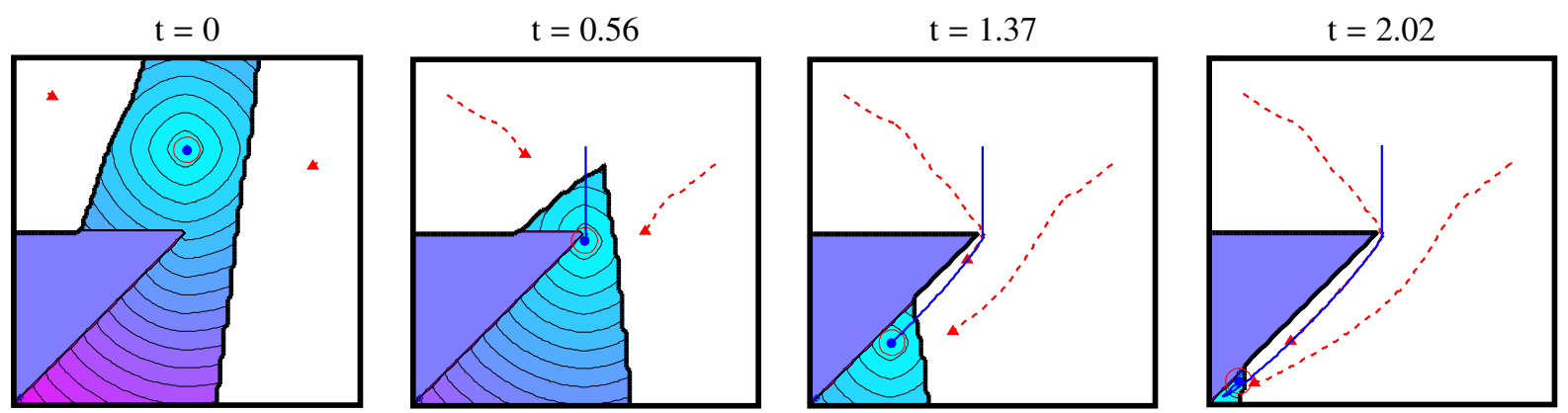

Figure 5: An example scenario showing 2 pursuers and 1 evader in a non-convex environment, solved using the modified FMM algorithm.

of trials against two other pursuit algorithms: pure-distance pursuit, where each pursuer instantaneously minimizes the distance between itself and the evader, and a numerical approximation of the optimal Hamilton-Jacobi-Isaacs solution on a grid. The pure-pursuit strategy was chosen for comparison purposes because it is straightforward to implement on arbitrarily defined game domains and is the optimal pursuit strategy for open domains with no boundaries. It is also guaranteed to result in capture for closed, simply connected domains [2]. Several simulation examples will be used to highlight some qualitative properties of the safe-reachable area-minimization pursuit strategy, and then the quantitative results of the numerical trials will be presented.

\subsection{Illustrative Examples}

A few simulations are presented here to highlight some qualitative aspects of the safe-reachable area-minimization pursuit algorithm. Pursuit in a non-convex environment is shown in Figure 5, which shows 2 pursuers (red triangles) pursuing a single evader (blue circle) in a simple, non-convex region with a triangular obstacle. The evader's safe-reachable set $\mathcal{S}_{e}$ is shown at each time, with equal time-to-reach contours plotted within $\mathcal{S}_{e}$. Convex domain simulations can be found in [17]. The simulations were conducted in Matlab on a Macbook Pro laptop with a $2.2 \mathrm{Ghz}$ Intel Core i7 processor with $8 \mathrm{~GB}$ of RAM, with computation per time-step of less than $1 \mathrm{~ms}$ to calculate inputs for all the pursuers in the analytic, convex case, and about $100 \mathrm{~ms}$ for each pursuer using the FMM algorithm.

\subsection{Comparison Tests}

The results of the comparison tests conducted to evaluate the safe-reachable area-minimization pursuit strategy will now be presented. Two groups of trials were conducted. The first set of trials matched safe-reachable area-minimization pursuit, puredistance pursuit, and the numerical HJI strategy against each other in tests with one pursuer and one evader. For these tests, a numerical approximation to the HJI equation was computed on a $40 \times 40$ grid for a simple non-convex region, shown in Figure 6(b), and the pursuer and evader strategies were evaluated by numerical differentiation. The three different pursuit strategies were then evaluated against the approximate optimal evader strategy for 500 initial conditions generated randomly.

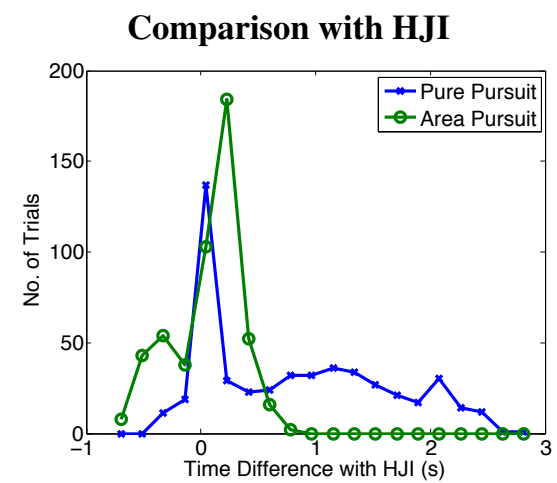

Figure 7: Histogram showing the difference in capture times in each single pursuer trial between the safe-reachable area minimization strategy, the pure-distance pursuit strategy, and the numerical Hamilton-Jacobi-Isaacs pursuit strategy.

The results of this test are displayed in Figure 7. In general, the area-minimization strategy performed slightly worse than the numerical HJI pursuit strategy, and the pure-distance pursuit strategy performed typically the worst among the three. However, it should be noted that the numerical HJI strategy depends on numerical differentiation of the approximated value function on a grid, and numerical errors can lead to sub-optimal pursuer and evader actions. For example, in $29 \%$ of trials the area minimization pursuit strategy out-performed the "optimal" HJI pursuer, and similarly in $8.5 \%$ of trials the pure-distance pursuit strategy resulted in faster capture-times.

In addition to the numerical issues discussed above, due to computational complexity, the HJI solution can only be found for the case of a single pursuer. To evaluate the performance of the pursuit strategy with multiple pursuers, a further series of tests were conducted comparing safe-reachable areaminimization pursuit with pure-distance pursuit. For these tests, the evader strategy was defined as the following: the evader selects as its target point $y^{*}$ the farthest point from itself in $\mathcal{S}_{e}$, that is

$$
y^{*}=\max _{y \in \mathcal{S}_{e}} \delta_{g}\left(y, x_{e}\right)
$$

where $\delta_{g}$ is the geodesic distance between $y$ and $x_{e}$. Once the evader reaches a certain distance (set here as half of the capture radius) from $y^{*}$, a new target is selected and the evader will proceed toward this target. 


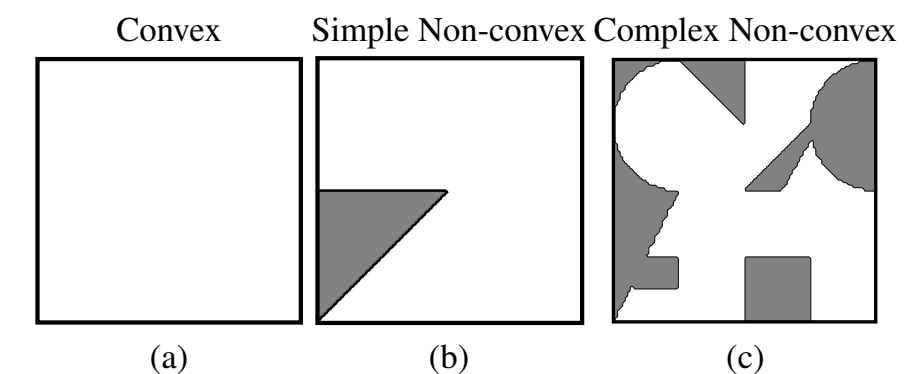

Figure 6: Domains used for the comparison simulations.

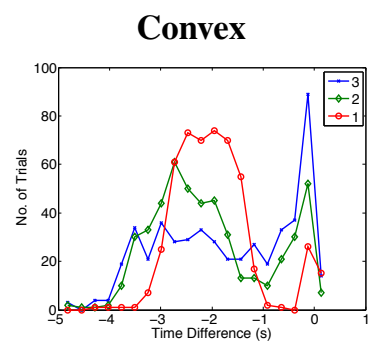

(a)

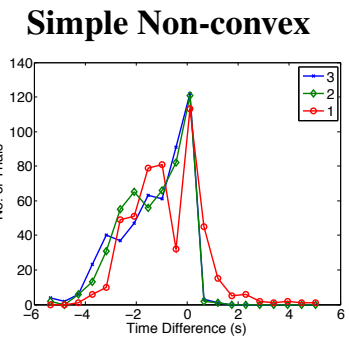

(b)

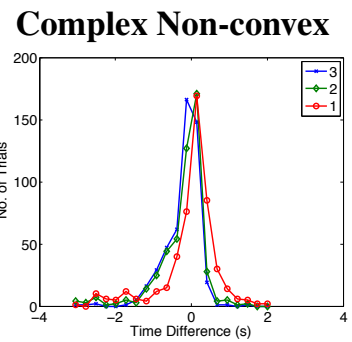

(c)

Figure 8: Histograms showing the number of trials versus difference in capture time between the pure-distance pursuit and safe-reachable area minimization strategies for (a) the convex game domain, (b) the simple non-convex domain, and (c) the complex non-convex domain.

Three sets of trials were conducted with 1,2 , and 3 pursuers, with one set in a square, convex region using the analytically derived pursuit strategy and two others in non-convex regions using FMM, shown in Figure 6. For each set, 500 random initial sets of pursuer and evader positions were generated. The results of the tests are summarized in Figure 8, showing histograms of the difference in capture-times between trials, defined as the capture-time required for the safe-reachable area strategy minus the capture-time required for pure-distance pursuit given a set of initial conditions.

Table 1 shows the fraction of trials in each case where the area pursuit strategy resulted in faster capture times than puredistance pursuit. Figure 8(a) shows the distribution of results in the convex environment. In this scenario, the safe-reachable area pursuit strategy resulted in clearly superior performance, with the vast majority of trials resulting in faster capture times. The distribution of times seems somewhat bimodal in these trials, with a number of trials where safe-reachable area minimization pursuit and pure-distance pursuit performed similarly, and then a large group where the safe-reachable area minimization pursuit strategy was clearly superior.

The results for the non-convex scenarios are shown in Figure 8(b) for the simple non-convex environment, and in Figure 8 (c) for the complex non-convex environment. The simple non-convex case still resulted in a large majority of trials where the safe-reachable area minimization pursuit strategy gave faster capture times, although in a smaller percentage of trials than the convex scenario. The complex non-convex case showed a decline in the performance of the safe-reachable area minimization strategy relative to the pure-distance pursuit strategy. This is due to the fact that the obstacles create areas where the width of the free space is of similar size to the capture ra- dius, thus the pure-distance pursuit can still "trap" the evader, lessening the advantage conferred by the safe-reachable area strategy. In fact, it is to be expected that as the space becomes more and more similar to a single long, narrow corridor, the pure-distance pursuit and area-pursuit strategies should have identical performance. This is especially evident in the trials with only 1 pursuer, where only $34 \%$ of trials resulted in faster capture with safe-reachable area-minimization pursuit, with a long tail of trials where the area pursuit performed much worse than pure-distance pursuit. These typically occurred in trials where the evader was able to escape from a confined portion of the game domain against the safe-reachable area minimizing pursuer, in part due to numerical errors in the area differentiation. Nonetheless, the safe-reachable area-minimization strategy showed a clear superiority in the trials with 2 and 3 pursuers, demonstrating the benefit of cooperation.

Table 2 shows the role played by cooperation in the Voronoibased cooperative pursuit. For each setup (number of pursuers and domain scenario), we again generated 500 random initial locations for the players as before. The first entry in each cell is the average capture time while the second entry is the standard deviation. We can see that in general, the decrease in capture time scales close to linear when there is few pursuers and scales sublinearly as the number of pursuers increases, something to be expected because the number of redundant pursuers (i.e., those do not share an active Voronoi boundary with the evader and hence are engaged in direct pursuit) is more likely to increase when there are more pursuers. Another trend to notice is that the variance is larger for the fewer players because the initial condition plays an important role in the capture time. As the number of players gets large, it is more likely that at least some pursuers will occupy advantageous positions relative to 


\begin{tabular}{|l|c|c|c|}
\hline & 1 pursuer & 2 pursuers & 3 pursuers \\
\hline \hline Convex & $91.6 \%$ & $98.2 \%$ & $96.6 \%$ \\
\hline Simple Non-convex & $67.2 \%$ & $86.2 \%$ & $86.4 \%$ \\
\hline Complex Non-convex & $34.0 \%$ & $52.8 \%$ & $60.4 \%$ \\
\hline
\end{tabular}

Table 1: Percentage of trials for which the safe-reachable area minimization pursuit strategy out-performed the pure-distance pursuit strategy.

\begin{tabular}{|l|c|c|c|}
\hline & Convex & Simple Non-convex & Complex Non-convex \\
\hline \hline 1 pursuer & $9.6 / 3.9$ & $6.4 / 2.7$ & $6.1 / 2.5$ \\
\hline 2 pursuers & $5.9 / 2.2$ & $3.5 / 1.5$ & $3.3 / 1.4$ \\
\hline 3 pursuers & $4.3 / 1.5$ & $2.2 / 1.0$ & $2.6 / 1.0$ \\
\hline 4 pursuers & $3.4 / 1.1$ & $1.8 / 0.8$ & $2.3 / 0.7$ \\
\hline 5 pursuers & $2.9 / 0.9$ & $1.5 / 0.5$ & $2.1 / 0.5$ \\
\hline
\end{tabular}

Table 2: Mean Capture Times (seconds) / Standard Deviation for the safereachable area minimization pursuit strategy.

the evader.

\section{Conclusions and Future Work}

The pursuit strategy presented in this work has several important strengths. The construction of the Voronoi partition or safereachable set allows a high-dimensional problem to be reduced to lower dimensions, easing the computational burden. Additionally, each pursuer can compute its inputs independently, allowing the strategy to run efficiently in real time. Yet information is shared through the partition itself, enabling cooperation between the pursuers and reducing capture time. The Voronoi partition also encompasses global information about the game domain, giving an advantage over strategies such as pure-distance pursuit that ignores the presence of obstacles and boundaries. Overall, safe-reachable area minimization seems to hold promise as a cooperative pursuit solution approach. As seen before in other works [7, 8, 16, 26], the visualization of the reachable set is a useful tool for human agents. Although GPS noise and time delay rendered the optimal headings sometimes unreliable in practice, the visualization of the set itself allowed the agents to compensate for these disturbances and head in the correct general direction to capture the evader. This result supports the idea that reachable sets are effective visual tools for assisting humans.

\section{References}

[1] M Aigner and M Fromme. A game of cops and robbers. Discrete Applied Mathematics, 8(1):1-12, 1984

[2] S Alexander, R Bishop, and R Ghrist. Pursuit and evasion in non-convex domains of arbitrary dimensions. In Proceedings of the Robotics: Science and Systems Conference, Philadelphia, Pennsylvania, August 2006.

[3] Brendan Ames, Andrew Beveridge, Rosalie Carlson, Claire Djang, Volkan Isler, Stephen Ragain, and Maxray Savage. A leapfrog strategy for pursuit-evasion in a polygonal environment. arXiv preprint arXiv:1401.2960, 2014.

[4] T. Başar and G. Olsder. Dynamic Noncooperative Game Theory. SIAM, Philadelphia, PA, 2 edition, 1999.
[5] E Bakolas and P Tsiotras. Optimal pursuit of moving targets using dynamic voronoi diagrams. Proceedings of the IEEE International Conference on Decision and Control, 2010.

[6] Deepak Bhadauria, Kyle Klein, Volkan Isler, and Subhash Suri. Capturing an evader in polygonal environments with obstacles: The full visibility case. Int. J. Rob. Res., 31(10):1176-1189, September 2012.

[7] M. Chen, Z. Zhou, and C. J. Tomlin. Multiplayer reach-avoid games via low dimensional solutions and maximum matching. In 2014 American Control Conference, pages 1444-1449, June 2014.

[8] M. Chen, Z. Zhou, and C. J. Tomlin. A path defense approach to the multiplayer reach-avoid game. In 53rd IEEE Conference on Decision and Control, pages 2420-2426, Dec 2014.

[9] W.A Cheung. Constrained pursuit-evasion problems in the plane. Master's thesis, Faculty of Graduate Studies, Computer Science, University of British Columbia, 2005

[10] Jerry Ding, Jonathan Sprinkle, S. Shankar Sastry, and Claire J. Tomlin. Reachability calculations for automated aerial refueling. In Proceedings of the IEEE International Conference on Decision and Control, pages 3706-3712, Cancun, Mexico, 2008.

[11] L. C. Evans and P. E. Souganidis. Differential games and representation formulas for solutions of Hamilton-Jacobi-Isaacs equations. Indiana University Mathematics Journal, 33(5):773-797, 1984.

[12] M. Falcone. Fast marching methods for front propagation. Lecture notes at "Introduction to Numerical Methods for Moving Boundaries", November 2007.

[13] M Falcone and R Ferretti. Semi-Lagrangian schemes for Hamilton-Jacobi equations, discrete representation formulae and Godunov methods. Journal of Computational Physics, 175(2):559-575, 2002.

[14] Brian P. Gerkey, Sebastian Thrun, and Geoff Gordon. Visibility-based pursuit-evasion with limited field of view. International Journal of Robotics Research, 25(4):299-315, 2006.

[15] Leonidas Guibas, Jean-Claude Latombe, Steven Lavalle, David Lin, and Rajeev Motwani. Visibility-based pursuit-evasion in a polygonal environment. In Frank Dehne, Andrew Rau-Chaplin, Jorg-Rudiger Sack, and Roberto Tamassia, editors, Algorithms and Data Structures, volume 1272 of Lecture Notes in Computer Science, pages 17-30. Springer Berlin / Heidelberg, 1997.

[16] H. Huang, J. Ding, W. Zhang, and C.J. Tomlin. A differential game approach to planning in adversarial scenarios: a case study on capture-theflag. In Proceedings of the IEEE International Conference on Robotics and Automation, Shanghai, China, 2011.

[17] Haomiao Huang, Wei Zhang, Jerry Ding, Dušan Stipanović, and C.J. Tomlin. Guaranteed decentralized pursuit-evasion in the plane with multiple pursuers. In Proceedings of the IEEE International Conference on Decision and Control, Orlando, Florida, 2011.

[18] R. Isaacs. Differential Games. Wiley, New York, 1967.

[19] S Kopparty and C.V Ravishankar. A framework for pursuit evasion games in $R^{n}$. Information Processing Letters, 96(3):114-122, 2005.

[20] S.M. LaValle and J.E. Hinrichsen. Visibility-based pursuit-evasion: the case of curved environments. IEEE Transactions on Robotics and Automation, 17(2):196 -202, April 2001.

[21] I.M. Mitchell, A.M. Bayen, and C.J. Tomlin. A time-dependent Hamilton-Jacobi formulation of reachable sets for continuous dynamic games. IEEE Transactions on Automatic Control, 50(7):947-957, 2005.

[22] T Parsons. Pursuit-evasion in a graph. Theory and Applications of Graphs, pages 426-441, 1978.

[23] J. A. Sethian. Fast marching methods. SIAM Review, 41(2):199-235, 1999.

[24] J. A. Sethian. Level set methods and fast marching methods, volume 3 of Cambridge Monographs on Applied and Computational Mathematics. Cambridge University Press, Cambridge, UK, second edition, 1999.

[25] Ryo Takei and Richard Tsai. Optimal trajectories of curvature constrained motion in the hamilton-jacobi formulation. J. Sci. Comput., 54(2-3):622644,2013

[26] Ryo Takei, Richard Tsai, Zhengyuan Zhou, and Yanina Landa. An efficient algorithm for a visibility-based surveillance-evasion game. Соттиnications in Mathematical Sciences, 7:1303-1327, 2012.

[27] Zhengyuan Zhou, Ryohei Takei, Haomiao Huang, and Claire J Tomlin. A general, open-loop formulation for reach-avoid games. In Decision and Control (CDC), 2012 IEEE 51st Annual Conference on, pages 65016506. IEEE, 2012. 